\title{
Controllability of Linear Dynamical Systems Under Input Sparsity Constraints
}

\author{
Geethu Joseph ${ }^{\circledR}$ and Chandra R. Murthy ${ }^{\circledR}$, Senior Member, IEEE
}

\begin{abstract}
In this article, we consider the controllability of a discrete-time linear dynamical system with sparse control inputs. Sparsity constraints on the input arises naturally in networked systems, where activating each input variable adds to the cost of control. We derive algebraic necessary and sufficient conditions for ensuring controllability of a system with an arbitrary transfer matrix. The derived conditions can be verified in polynomial time complexity, unlike the more traditional Kalman-type rank tests. Further, we characterize the minimum number of input vectors required to satisfy the derived conditions for controllability. Finally, we present a generalized Kalman decomposition-like procedure that separates the state-space into subspaces corresponding to sparse-controllable and sparse-uncontrollable parts. These results form a theoretical basis for designing networked linear control systems with sparse inputs.
\end{abstract}

Index Terms - Controllability, Kalman rank test, linear dynamical systems, Popov-Belevitch-Hautus (PBH) test, sparsity, switched linear systems.

\section{INTRODUCTION}

In networked control systems, the notion of controllability refers to the ability to drive the system from an arbitrary initial state to a desired final state in a finite amount of time. Complete characterization of controllability of linear dynamical systems using unconstrained inputs have pure algebraic rank-based forms, and are rather easily verifiable [1], [2]. However, in applications involving networked control systems, it is often necessary to select a small subset of the available sensors or actuators at each time instant due to communication bandwidth, cost, or energy constraints [3], [4]. Further, it is often desirable to select a different subset of nodes at each time instant to improve the network lifetime [5]. Now, when the number of actuators or input variables that can be activated at each time instant is limited, the system may become uncontrollable because all the feasible control signals are restricted to lie in the union of low-dimensional subspaces. The controllability of linear dynamical systems under sparse input constraints is the focus of this article.

Manuscript received December 29, 2019; accepted April 8, 2020 Date of publication April 20, 2020; date of current version January 28 , 2021. The work of Geethu Joseph was supported by the Intel India Ph.D. fellowship, and the work of Chandra R. Murthy was supported by the MeitY Young Faculty Research Fellowship. Recommended by Associate Editor S. Azuma. (Corresponding author: Geethu Joseph.)

Geethu Joseph was with the Department of Electrical Communication Engineering, Indian Institute of Science, Bangalore 560012, India She is now with the Department of Electrical Engineering and Computer Science, Syracuse University, Syracuse, NY 13244 USA (e-mail: gjoseph@syr.edu).

Chandra R. Murthy is with the Department of Electrical Communication Engineering, Indian Institute of Science, Bangalore 560012, India (e-mail: cmurthy@iisc.ac.in).

Digital Object Identifier 10.1109/TAC.2020.2989245

\section{A. Related Literature}

We discuss the relationship between the problem considered in this article and the existing literature in control theory and sparse signal processing.

1) Time-Varying Actuator Scheduling Problem: This problem focuses on finding a schedule for sparse actuator control, such that the system is sparse-controllable [5]-[7]. These works rely on a well-known condition for controllability-namely, rank of the Gramian matrix of the sparsity-constrained system. However, finding a sequence of control inputs that satisfy the rank condition on the Gramian matrix is an NP-hard combinatorial problem [8], [9]. Hence, different quantitative measures of controllability based on the Gramian matrix have been considered-smallest eigenvalue, the trace of the inverse, the inverse of the trace, the determinant, maximum diagonal element, etc. [5]. More importantly, these studies do not directly address the question of whether or not the system can be controlled by sparse inputs.

2) Minimal Input Selection Problem: The minimal input selection involves selecting a small set of input variables so that the system is controllable using the selected set [8]-[10]. This problem imposes an extra constraint that the support of the control input remains unchanged for all time instants. We discuss and contrast the two cases in detail in Section III-B.

3) Design of Sparse Control Inputs: Some work connecting compressive sensing and control theory focus on the design (recovery) of sparse control inputs using a limited number of observations [11] [13]. These studies assume the existence of a set of sparse control inputs that can drive a given initial state to a desired final state.

4) Observability Under Sparsity Constraints: Recently, the observability of linear systems with a sparse initial state has also been studied [14], [15]. However, our problem assumes a general initial state and sparse control inputs. Therefore, the problems have different sparsity models, and consequently, require separate analysis.

\section{B. Our Contributions}

In this article, we answer the following key questions.

Q1) What are necessary and sufficient conditions for ensuring controllability under sparse input constraints? Can we devise a computationally simple test for controllability?

Q2) If a system is controllable using sparse inputs, how many control input vectors needed to drive the system from a given initial state to an arbitrary final state?

Q3) If the system is not controllable using sparse inputs, what part of the state space is reachable using sparse inputs?

Answering the above questions requires a fresh look at controllability, and we start by deriving a Popov-Belevitch-Hautus (PBH)-like test [2], which, unlike the Gramian matrix-based tests, allows one to check for sparse-controllability of a system without solving a combinatorial problem. Our specific contributions are as follows. 
1) We establish a set of the necessary and sufficient conditions for the controllability of a linear system under sparse inputs in Section III. Using these conditions, we present a simple procedure to check the controllability of any system using sparse inputs.

2) We upper and lower bound the minimum number of input vectors that can steer the system from any given initial state to any desired final state in Section IV. We show that the upper bound is no more than the length of the state vector, which is also an upper bound for the minimum number of input vectors for an unconstrained system.

3) We present a procedure to convert a representation of any linear dynamical system into a standard form in Section V. The standard form separates the state-space into uncontrollable, sparseuncontrollable, and sparse-controllable components.

In a nutshell, this article presents new results on the controllability of linear dynamical systems under sparsity constraints on the input. We also note that the classical results for the unconstrained system can be recovered as a special case of our results, by relaxing the sparsity constraint. A longer version of this article, which contains additional results and examples, can be found in [16].

Notation: In the sequel, we use $|\cdot|$ to denote the cardinality of a set and $\|\cdot\|_{0}$ to denote the $\ell_{0}$ norm of a vector. For any positive integer $a,[a]$ denotes the set $\{1,2, \ldots, a\}$. The symbols $\boldsymbol{I}$ and $\mathbf{0}$ represent the identity matrix and the all zero matrix (or vector), respectively. The notation $\boldsymbol{A}_{i}$ denotes the $i$ th column of the matrix $\boldsymbol{A}$, and $\boldsymbol{A}_{\mathcal{S}}$ represents the submatrix of $\boldsymbol{A}$ formed by the columns indexed by the set $\mathcal{S}$. Also, $\mathcal{C} \mathcal{S}\{\cdot\}, \operatorname{Rank}\{\cdot\}$ and $(\cdot)^{\top}$ represent the column space, rank, and transpose of a matrix, respectively.

\section{SYSTEM MODEL}

We consider the discrete-time linear dynamical system whose state at time $k$, denoted by $\boldsymbol{x}_{k} \in \mathbb{R}^{N}$, evolves as

$$
\boldsymbol{x}_{k}=\boldsymbol{D} \boldsymbol{x}_{k-1}+\boldsymbol{H} \boldsymbol{h}_{k}
$$

where the transfer matrix $\boldsymbol{D} \in \mathbb{R}^{N \times N}$ and input matrix $\boldsymbol{H} \in \mathbb{R}^{N \times L}$. Here, the input vectors $\boldsymbol{h}_{k} \in \mathbb{R}^{L}$ are assumed to be sparse, i.e., $\left\|\boldsymbol{h}_{k}\right\|_{0} \leq s$, for all values of $k$. We denote the rank of the matrices $\boldsymbol{D}$ and $\boldsymbol{H}$ using $R_{\boldsymbol{D}}$ and $R_{\boldsymbol{H}}$, respectively.

We formally define the notion of controllability using sparse inputs as follows.

Definition 1 (Sparse-Controllability): The system in (1) is said to be $s$-sparse-controllable if, for any initial state $\boldsymbol{x}_{0}=\boldsymbol{x}_{\text {init }}$ and any final state $\boldsymbol{x}_{\text {final }}$, there exists inputs $\left\{\boldsymbol{h}_{k}\right\}_{k=1}^{K}$ such that $\left\|\boldsymbol{h}_{k}\right\|_{0} \leq s$, which steers the system from the state $\boldsymbol{x}_{0}=\boldsymbol{x}_{\text {init }}$ to $\boldsymbol{x}_{K}=\boldsymbol{x}_{\text {final }}$ for some finite $K$

Next, to characterize the sparse-controllability of the system, we consider the following equivalent system of equations:

$$
\boldsymbol{x}_{K}-\boldsymbol{D}^{K} \boldsymbol{x}_{0}=\tilde{\boldsymbol{H}}_{(K)} \boldsymbol{h}_{(K)}
$$

where we define the matrices as follows:

$$
\begin{aligned}
\tilde{\boldsymbol{H}}_{(K)} & =\left[\begin{array}{llll}
\boldsymbol{D}^{K-1} \boldsymbol{H} & \boldsymbol{D}^{K-2} \boldsymbol{H} & \ldots \boldsymbol{H}
\end{array}\right] \in \mathbb{R}^{N \times K L} \\
\boldsymbol{h}_{(K)} & =\left[\begin{array}{llll}
\boldsymbol{h}_{1}^{\top} & \boldsymbol{h}_{2}^{\top} & \ldots & \boldsymbol{h}_{K}^{\top}
\end{array}\right]^{\top} \in \mathbb{R}^{K L} .
\end{aligned}
$$

Note that $\boldsymbol{h}_{(K)}$ is a piecewise sparse vector formed by concatenating $K$ vectors, each with sparsity at most $s$.

\section{Necessary and Sufficient Conditions For SPARSE-CONTROLLABILITY}

This section addresses question Q1 in Section I. Now, it is known that the system is sparse-controllable if, for some finite $K$, there exist index sets $\left\{\mathcal{S}_{i}\right\}_{i=1}^{K}, \mathcal{S}_{i} \subseteq\{1,2, \ldots, L\},\left|\mathcal{S}_{i}\right|=s$, for $i=1,2, \ldots, K$, such that the following submatrix of $\tilde{\boldsymbol{H}}_{(K)}$ has rank $N$ :

$$
\left[\begin{array}{llll}
\boldsymbol{D}^{K-1} \boldsymbol{H}_{\mathcal{S}_{1}} & \boldsymbol{D}^{K-2} \boldsymbol{H}_{\mathcal{S}_{2}} & \cdots & \boldsymbol{H}_{\mathcal{S}_{K}}
\end{array}\right] \in \mathbb{R}^{N \times K s} .
$$

In the sequel, we refer this condition to as the Kalman-type rank test. Note that the first $(K-1) N$ columns of $\tilde{\boldsymbol{H}}_{(K)}$ belong to $\mathcal{C S}\{\boldsymbol{D}\}$. Hence, to satisfy the Kalman-type rank test, $\mathcal{S}_{K}$ should be such that $\mathcal{C S}\left\{\boldsymbol{H}_{\mathcal{S}_{K}}\right\}$ should contain the left null space of $\boldsymbol{D}$. Thus, a necessary condition for sparse-controllability is the existence of an index set $\mathcal{S}$ with $s$ entries such that $\operatorname{Rank}\left\{\left[\begin{array}{ll}\boldsymbol{D} & \boldsymbol{H}_{\mathcal{S}}\end{array}\right]\right\}=N$, which is possible only if $s \geq N-R_{D}$. Further, a system can be sparse-controllable only if it is controllable using unconstrained inputs. Therefore, for sparse-controllability, it is necessary that the system is controllable and $s \geq N-R_{D}$. In fact, these two conditions are not only necessary but also sufficient, as we show in the following theorem.

Theorem 1: The system in (1) is $s$-sparse-controllable if and only if $\operatorname{Rank}\{[\lambda \boldsymbol{I}-\boldsymbol{D} \quad \boldsymbol{H}]\}=N \leq s+R_{\boldsymbol{D}}$ for all $\lambda \in \mathbb{C}$.

Proof: See Appendix A.

Note that there are two separate conditions here: one, a condition on the rank of the matrix $[\lambda \boldsymbol{I}-\boldsymbol{D} \quad \boldsymbol{H}] \in \mathbb{R}^{N+L}$, which we refer to as the rank condition of Theorem 1; and two, a lower bound on the sparsity $s$, which we refer to as the inequality condition of Theorem 1. The rank condition is same as the classical PBH test [2] which is independent of the sparsity level $s$, while the inequality condition is independent of the input matrix $\boldsymbol{H}$. We make the following remarks.

1) A reversible system, i.e., a system with an invertible state transition matrix $\boldsymbol{D}$, is $s$-sparse-controllable for any $0<s \leq L$ if and only if it is controllable. Similarly, when $L=1$, the notion of sparsecontrollability and controllability are the same, and hence Theorem 1 reduces to the $\mathrm{PBH}$ test.

2) If the system defined by the matrix pair $\left(\boldsymbol{D}, \boldsymbol{H}_{\mathcal{S}}\right)$ is controllable for some index set $\mathcal{S}$ with $s$ entries, the system is $s$-sparse-controllable. In particular, a controllable system with $R_{\boldsymbol{H}} \leq s$ is $s$-sparsecontrollable.

3) The system given by (1) is controllable using inputs that are $s$-sparse under a basis $\Psi \in \mathbb{R}^{L \times L}$ if and only if the system is controllable using inputs that are $s$-sparse under the canonical basis. This follows by replacing $\boldsymbol{H}$ with $\boldsymbol{H} \boldsymbol{\Psi}$ in Theorem 1, and noting that for any $\lambda \in \mathbb{C}$

$$
\operatorname{Rank}\{[\lambda \boldsymbol{I}-\boldsymbol{D} \quad \boldsymbol{H} \boldsymbol{\Psi}]\}=\operatorname{Rank}\left\{\left[\begin{array}{ll}
\lambda \boldsymbol{I}-\boldsymbol{D} & \boldsymbol{H}
\end{array}\right]\right\} .
$$

4) The verification of sparse-controllability has the same complexity as the classical PBH test. This is because, we only need to additionally check the inequality in Theorem 1 , and $R_{D}$ is already known from the PBH test. Thus, Theorem 1 allows us to verify the controllability of any discrete system in polynomial complexity in $N$, independent of the sparsity $s$. On the other hand, to verify the Kalman-type rank test, we need to perform $\left(\begin{array}{l}L \\ s\end{array}\right)^{N}$ rank computations. Further, since the Kalman-type rank test involves powers of $D$, numerical stability also needs to be considered.

\section{A. Output Controllability}

We consider the linear dynamical system described by (1) and the following output relation:

$$
\boldsymbol{y}_{k}=\boldsymbol{A} \boldsymbol{x}_{k}
$$


where the output matrix $\boldsymbol{A} \in \mathbb{R}^{m \times N}$ with $m<N$. Similar to Definition 1, we define the notion of output s-sparse-controllability as the existence of an $s$-sparse sequence of inputs which steers the system from initial state $\boldsymbol{x}_{0}$ to a final output $\boldsymbol{y}_{K}$, for some finite $K$. Now, to characterize the output sparse-controllability, we consider the following equivalent system of equations:

$$
\boldsymbol{y}_{K}-\boldsymbol{A} \boldsymbol{D}^{K} \boldsymbol{x}_{0}=\boldsymbol{A} \tilde{\boldsymbol{H}}_{(K)} \boldsymbol{h}_{(K)} .
$$

In [17], a Kalman test for output controllability of an unconstrained system is derived, which states that the system given by (1) and (6) is output controllable if and only if the matrix $\boldsymbol{A} \tilde{\boldsymbol{H}}_{(K)}$ has full row rank for some finite $K$. However, a direct extension of this result to the case of output sparse-controllability leads to a computationally expensive combinatorial test as follows. The system is output controllable if and only if, for some finite $K$, there exists a submatrix of $\boldsymbol{A} \tilde{\boldsymbol{H}}_{(K)}$ with rank $m$ of the form

$$
\boldsymbol{A}\left[\begin{array}{llll}
\boldsymbol{D}^{K-1} \boldsymbol{H}_{\mathcal{S}_{1}} & \boldsymbol{D}^{K-2} \boldsymbol{H}_{\mathcal{S}_{2}} & \ldots & \boldsymbol{H}_{\mathcal{S}_{K}}
\end{array}\right] \in \mathbb{R}^{m \times K s}
$$

such that the index set $\mathcal{S}_{i} \subseteq\{1,2, \ldots, L\}$ and $\left|\mathcal{S}_{i}\right|=s$, for $i=$ $1,2, \ldots, K$. Hence, we first present the following PBH test-type result for output (unconstrained) controllability:

Proposition 1: For an unconstrained system given by (1) and (6), the system is output controllable only if, for all $\lambda \in \mathbb{C}$, the rank of $\boldsymbol{A}[\lambda \boldsymbol{I}-\boldsymbol{D} \quad \boldsymbol{H}] \in \mathbb{R}^{m \times(N+L)}$ is $m$.

Proof: Our proof is by contradiction. Suppose that, for some $\lambda \in \mathbb{C}$, the matrix $\boldsymbol{A}[\lambda \boldsymbol{I}-\boldsymbol{D} \quad \boldsymbol{H}]$ does not have full row rank. Then, there exists a $\mathbf{0} \neq \boldsymbol{z} \in \mathbb{C}^{m}$ such that

$$
\boldsymbol{z}^{\top} \boldsymbol{A D}=\lambda \boldsymbol{z}^{\top} \boldsymbol{A} \text { and } \boldsymbol{z}^{\top} \boldsymbol{A} \boldsymbol{H}=\mathbf{0}
$$

which implies $\boldsymbol{z}^{\top} \boldsymbol{A} \tilde{\boldsymbol{H}}_{(K)}=\mathbf{0}$ for all $K$. Hence, the Kalman test is violated, and the system is not output controllable.

Our extension of Theorem 1 to output sparse-controllability is as follows

Corollary 1: The system given by (1) and (6) is output $s$-sparsecontrollable only if $s \geq m-\operatorname{Rank}\{\boldsymbol{A} \boldsymbol{D}\}$, and for all $\lambda \in \mathbb{C}$, the rank of $\boldsymbol{A}[\lambda \boldsymbol{I}-\boldsymbol{D} \quad \boldsymbol{H}] \in \mathbb{R}^{m \times(N+L)}$ is $m$.

Proof: The proof is similar to that of Theorem 1 in Appendix A. We replace $\boldsymbol{z}$ in the last part of the proof with $\boldsymbol{A} \boldsymbol{z}$ to show the necessity of the above conditions.

Corollary 1 is the same as Theorem 1, except for a premultiplication with $\boldsymbol{A}$. We make the following observations.

1) We note that $\operatorname{Rank}\left\{\boldsymbol{A} \boldsymbol{H}^{*}\right\} \leq \operatorname{Rank}\{\boldsymbol{A}\}$ for any matrix $\boldsymbol{H}^{*}$. Hence, if $\operatorname{Rank}\{\boldsymbol{A}\}<m$, the Kalman test fails and the system is not output sparse-controllable.

2) Suppose $\operatorname{Rank}\{\boldsymbol{A}\}=m$ for an $s$-sparse-controllable system. Invoking Sylvester's rank inequality [18], we get

$$
\begin{aligned}
m=\operatorname{Rank}\{\boldsymbol{A}\}+ & \operatorname{Rank}\left\{\boldsymbol{H}^{*}\right\}-N \\
& \leq \operatorname{Rank}\left\{\boldsymbol{A} \boldsymbol{H}^{*}\right\} \leq \operatorname{Rank}\{\boldsymbol{A}\}=m
\end{aligned}
$$

where $\boldsymbol{H}^{*} \in \mathbb{R}^{N \times K s}$ is the submatrix of $\tilde{\boldsymbol{H}}_{(K)}$ that satisfies the Kalman test for state sparse-controllability, for some finite $K$. Hence, the system is output $s$-sparse-controllable. Therefore, when $\operatorname{Rank}\{\boldsymbol{A}\}=m$, the conditions in Corollary 1 are less restrictive than those in Theorem 1 , as the output dimension $m \leq N$.

\section{B. Inputs With Common Support}

We recall the minimal input selection problem discussed in Section I. For such a problem, the system is controlled using sparse inputs with a common support, i.e., when the indices of the nonzero entries of all the inputs coincide. In this case, the effective system has the transfer matrix-input matrix pair as $\left(\boldsymbol{D}, \boldsymbol{H}_{\mathcal{S}}\right)$ for some index set $\mathcal{S}$ such that $|\mathcal{S}|=s$. Hence, the controllability conditions are given as follows.

i) For some finite positive integer $K$, there exists a $N \times K s$ subma$\operatorname{trix}\left[\boldsymbol{D}^{K-1} \boldsymbol{H}_{\mathcal{S}} \boldsymbol{D}^{K-2} \boldsymbol{H}_{\mathcal{S}} \ldots \boldsymbol{H}_{\mathcal{S}}\right]$ of $\tilde{\boldsymbol{H}}_{(K)}$ with rank $N$, where $\mathcal{S} \subseteq[L]$ and $|\mathcal{S}|=s$.

ii) For all $\lambda \in \mathbb{C}$, the rank of $\left[\lambda \boldsymbol{I}-\boldsymbol{D} \quad \boldsymbol{H}_{\mathcal{S}}\right] \in \mathbb{R}^{N \times(N+s)}$ is $N$, for some $\mathcal{S} \subseteq\{1,2, \ldots, L\}$ such that $|\mathcal{S}|=s$.

Clearly, (ii) above implies the two conditions of Theorem 1. Therefore, the above conditions are more stringent than those in Theorem 1, which is expected due to the additional requirement of using a common support. Thus, a system with sparse inputs with time-varying support offers greater flexibility and control, and incurs a similar communication cost, ${ }^{1}$ compared to a system that uses sparse inputs with a common support.

From the PBH-type condition, $s$-sparse-controllability with a common support holds only if

$$
\min \left\{R_{\boldsymbol{H}}, s\right\} \geq g_{\boldsymbol{D}} \geq N-R_{\boldsymbol{D}}
$$

where $g_{D}$ is the largest geometric multiplicity of an eigenvalue of $\boldsymbol{D}$.

To sum up, in this section, we answered Q1 posed in Section I. We address the question Q2 in the following section.

\section{Minimum Number of CONTROL InPUt Vectors}

In this section, we bound the minimum number of input vectors that are required to drive the system from any given state to any final state. For comparison, we first state the corresponding result for the unconstrained system. In this section, $q$ denotes the degree of the minimal polynomial of $\boldsymbol{D}$.

Theorem 2: For a controllable system, the minimum number of input vectors $K$ required to steer the system from any given state to any other state satisfies

$$
N / R_{\boldsymbol{H}} \leq K \leq \min \left\{q, N-R_{\boldsymbol{H}}+1\right\} \leq N .
$$

Proof: See [19, Sec. 6.2.1]

We note that when we restrict the admissible inputs to sparse vectors, the minimum number of input vectors required can increase. This change is captured by the following theorem.

Theorem 3: For an $s$-sparse-controllable system, the minimum number of $s$-sparse input vectors $K^{*}$ required to steer the system from any given state to any other state satisfies

$$
\frac{N}{R_{\boldsymbol{H}, s}^{*}} \leq K^{*} \leq \min \left\{q\left\lceil\frac{S^{*}}{s}\right\rceil, N-R_{\boldsymbol{H}, s}^{*}+1\right\} \leq N
$$

where $R_{\boldsymbol{H}, s}^{*} \triangleq \min \left\{R_{\boldsymbol{H}}, s\right\}$ and

$$
\begin{aligned}
& S^{*} \triangleq \min \{T: T=|\mathcal{S}| \text { for } \mathcal{S} \subseteq[L] \\
& \text { and } \left.\operatorname{Rank}\left\{\left[\begin{array}{ll}
\boldsymbol{D}-\lambda \boldsymbol{I} & \boldsymbol{H}_{\mathcal{S}}
\end{array}\right]\right\}=N, \forall \lambda \in \mathbb{C}\right\} \text {. }
\end{aligned}
$$

Proof: See Appendix B.

The above result can be intuitively explained as follows. At each time instant, we use at most $s$ linearly independent columns of $\boldsymbol{H}$ to drive the system. Therefore, $R_{H}$ is replaced with $R_{H, s}^{*}$. Also, the first term of the upper bound is computed by mapping the system to the reduced controllable system $\left(\boldsymbol{D}, \boldsymbol{H}_{\mathcal{S}^{*}}\right)$. The reduced system retains the least number of columns of $\boldsymbol{H}$ that are necessary to ensure controllability. Thus, under sparse inputs, we need $\left[\left|\mathcal{S}^{*}\right| / s\right\rceil$ times larger number of inputs compared to an unconstrained system. We make the following further observations from Theorem 3 .

\footnotetext{
${ }^{1}$ The communication cost remains of order $s$, since the support can be conveyed using $s \log (L)$ bits.
} 
1) Using the fact that that $S^{*} \leq R_{H}$, and from Theorem 1 which implies $R_{H, s}^{*} \geq \max \left\{N-R_{D}, 1\right\}$, we can get a relaxed bound instead of (12) as follows:

$$
\frac{N}{\min \left\{R_{\boldsymbol{H}}, s\right\}} \leq K^{*} \leq \min \left\{q\left\lceil\frac{R_{\boldsymbol{H}}}{s}\right\rceil, R_{\boldsymbol{D}}+1, N\right\} .
$$

2) The bound is invariant under right or left multiplication of $\boldsymbol{H}$ by a nonsingular matrix, and under any similarity transform on $\boldsymbol{D}$.

3) As $s$ increases, the system has more flexibility, and thus requires fewer number of input vectors to ensure controllability. Hence, the fact that the bounds are nonincreasing in $s$ is intuitively satisfying.

4) The upper and lower bounds in Theorem 3 meet when $N / R_{H, s}^{*}=$ $N-R_{\boldsymbol{H}, s}^{*}+1$, which gives $R_{\boldsymbol{H}, s}^{*}$ as 1 or $N$. Similarly, for $s=1$, the lower and upper bounds in Theorem 3 are equal, and $K^{*}=N$. Further, if $R_{\boldsymbol{H}} \geq s$, we get $R_{\boldsymbol{H}, s}^{*}=s$, and thus the bounds are equal when $s=N$.

5) We consider three cases for comparison with Theorem 2.

a) When $s=L$, which corresponds to the unconstrained case, Theorem 3 reduces to Theorem 2 , as expected.

b) When $s \geq S^{*} \geq R_{H}$, Theorem 3 reduces to Theorem 2, as $R_{\boldsymbol{H}, s}^{*}=R_{\boldsymbol{H}}$. This follows because when $s \geq R_{\boldsymbol{H}}$, $\mathcal{C S}\left\{\tilde{\boldsymbol{H}}_{(K)}\right\}$ is the same as the column space of an $N \times K s$ submatrix of $\tilde{\boldsymbol{H}}_{(K)}$ with maximum rank.

c) When $\min \left\{q, N-\boldsymbol{R}_{\boldsymbol{H}}+1\right\}=N$, the system requires the same number of inputs to achieve controllability and $s$ sparse-controllability, for any $s$. However, this is possible only if $R_{\boldsymbol{H}}=1$. When $s \geq R_{\boldsymbol{H}}$, the system is equivalent to an unconstrained system, as discussed above.

The following interesting corollary bounds the number of $s$-sparse input vectors that ensures output controllability.

Corollary 2: For an output $s$-sparse-controllable system, the minimum number of input vectors $K^{*}$ required to steer any initial output to any final output satisfies

$$
\frac{m}{R_{\boldsymbol{A H}, s}^{*}} \leq K^{*} \leq \min \left\{q\left\lceil\frac{R_{\boldsymbol{H}}}{s}\right\rceil, m-R_{\boldsymbol{A H}, s}^{*}+1\right\} \leq m
$$

where $R_{\boldsymbol{A} \boldsymbol{H}, s}^{*}=\min \{\operatorname{Rank}\{\boldsymbol{A} \boldsymbol{H}\}, s\}$.

The bounds in Corollary 2 are smaller than those in Theorem 3, because the dimension of the output space, $m$, is smaller than that of the state space, $N$. Further, substituting $s=L$ in Corollary 2, we see that for an output controllable system, the minimum number of input vectors $K$ required to steer any initial output to any final output satisfies

$$
\frac{m}{\operatorname{Rank}\{\boldsymbol{A} \boldsymbol{H}\}} \leq K \leq \min \{q, m-\operatorname{Rank}\{\boldsymbol{A} \boldsymbol{H}\}+1\} \leq m .
$$

Similarly, we can extend Theorem 3 to the common support case discussed in Section III-B.

Corollary 3: For a system that is controllable using $s$-sparse inputs with a common support, if $R_{\boldsymbol{H}, s}^{*}=\min \left\{R_{\boldsymbol{H}}, s\right\}$, the minimum number of input vectors $K^{*}$ required to steer any initial output to any final output satisfies

$$
\frac{N}{R_{\boldsymbol{H}, s}^{*}} \leq K^{*} \leq \min \left\{q, N-R_{\boldsymbol{H}, s}^{*}+1\right\} \leq N .
$$

Proof: The proof follows from Theorem 2 and the fact that there exists an index set $\mathcal{S} \subseteq[L]$ such that $|\mathcal{S}|=s$ and the system defined by $\left(\boldsymbol{D}, \boldsymbol{H}_{\mathcal{S}}\right)$ is controllable.

\section{Decomposing Sparse-Controllable States}

In this section, we consider Q3 in Section I, and present a decomposition of the state space into sparse-controllable, sparse-uncontrollable, and uncontrollable subspaces. We begin with the observation that $s$-sparse-controllability inherits the invariance under a change of basis property of the conventional controllability, as discussed in the proposition below.

Proposition 2 (Invariance under change of basis): The system defined by the matrix pair $(\boldsymbol{D}, \boldsymbol{H})$ is $s$-sparse-controllable if and only if the system defined by $\left(\boldsymbol{U}^{-1} \boldsymbol{D} \boldsymbol{U}, \boldsymbol{U}^{-1} \boldsymbol{H}\right)$ is $s$-sparse-controllable for every nonsingular $U \in \mathbb{R}^{N \times N}$.

Proof: We note that when $\boldsymbol{D}$ and $\boldsymbol{H}$ are replaced with $\boldsymbol{U}^{-1} \boldsymbol{D} \boldsymbol{U}$ and $\boldsymbol{U}^{-1} \boldsymbol{H}$ respectively, in (3), we get $\boldsymbol{U}^{-1} \tilde{\boldsymbol{H}}_{(K)}$ instead of $\tilde{\boldsymbol{H}}_{(K)}$. Now, the result follows from the Kalman-type rank test and the fact every submatrix of $\tilde{\boldsymbol{H}}_{(K)}$ and $\boldsymbol{U}^{-1} \tilde{\boldsymbol{H}}_{(K)}$ have the same rank.

Inspired by the above proposition and in the same spirit as the Kalman decomposition [20], we transform the original system to an equivalent standard form using a change of basis, such that the transformed state-space is separated into an $s$-sparse-controllable subspace and an orthogonal $s$-sparse-uncontrollable subspace. To this end, we first separate the controllable and uncontrollable states using the Kalman decomposition. Next, we identify the sparse-controllable part of the controllable part, for which we use the inequality condition of Theorem 1. For this, we find a basis for the controllable part such that the transformed state-space separates into two subsystems: one which satisfies the inequality condition, and the other which does not. We now formally present the procedure for the decomposition, followed by an explanation of why the procedure works. This procedure assumes that $\operatorname{Rank}\{\boldsymbol{D}\}=\operatorname{Rank}\left\{\boldsymbol{D}^{2}\right\}$.

1) Find a basis for $\mathcal{C S}\left\{\tilde{\boldsymbol{H}}_{(N)}\right\}$ as $\left\{\boldsymbol{u}_{i}\right\}_{i=1}^{R}$, where $R \leq N$ is the rank of $\tilde{\boldsymbol{H}}_{(N)}$. Extend the basis by adding $N-R$ linearly independent vectors $\left\{\boldsymbol{u}_{i}\right\}_{i=R+1}^{N}$ to define the invertible matrix $\boldsymbol{U} \triangleq$ $\left[\boldsymbol{u}_{1} \boldsymbol{u}_{2}, \ldots, \boldsymbol{u}_{N}\right] \in \mathbb{R}^{N \times N}$.

2) Compute $\check{\boldsymbol{D}}=\boldsymbol{U}^{-1} \boldsymbol{D} \boldsymbol{U} \in \mathbb{R}^{N \times N}$ and $\check{\boldsymbol{H}}=\boldsymbol{U}^{-1} \boldsymbol{H} \in \mathbb{R}^{N \times L}$ which take the following forms:

$$
\check{\boldsymbol{D}}=\left[\begin{array}{cc}
\check{\boldsymbol{D}}_{(1)} & \check{\boldsymbol{D}}_{(2)} \\
\mathbf{0} & \check{\boldsymbol{D}}_{(3)}
\end{array}\right] \quad \check{\boldsymbol{H}}=\left[\begin{array}{c}
\check{\boldsymbol{H}}_{(1)} \\
\mathbf{0}
\end{array}\right]
$$

where $\check{\boldsymbol{D}}_{(1)} \in \mathbb{R}^{R \times R}$ and $\check{\boldsymbol{H}}_{(1)} \in \mathbb{R}^{R \times L}$.

3) Use the Jordan decomposition to get the following:

$$
\check{\boldsymbol{D}}_{(1)}=\boldsymbol{V}\left[\begin{array}{cc}
\overline{\boldsymbol{D}}_{(11)} \in \mathbb{R}^{r \times r} & \mathbf{0} \\
\mathbf{0} & \mathbf{0}
\end{array}\right] \boldsymbol{V}^{-1}
$$

where $\boldsymbol{V} \in \mathbb{R}^{R \times R}$ and $r \leq R$ is the rank of $\check{\boldsymbol{D}}_{(1)}$.

4) Define an invertible matrix $\boldsymbol{W} \in \mathbb{R}^{N \times N}$ as follows:

$$
\boldsymbol{W} \triangleq\left[\begin{array}{cc}
\boldsymbol{V} \in \mathbb{R}^{R \times R} & \mathbf{0} \in \mathbb{R}^{R \times N-R} \\
\mathbf{0} \in \mathbb{R}^{N-R \times R} & \boldsymbol{I} \in \mathbb{R}^{N-R \times N-R}
\end{array}\right] .
$$

5) Compute $\quad \overline{\boldsymbol{D}}=\boldsymbol{W}^{-1} \check{\boldsymbol{D}} \boldsymbol{W} \in \mathbb{R}^{N \times N} \quad$ and $\quad \overline{\boldsymbol{H}}=\boldsymbol{W}^{-1} \check{\boldsymbol{H}} \in$ $\mathbb{R}^{N \times L}$, which take the following forms:

$$
\overline{\boldsymbol{D}}=\left[\begin{array}{ccc}
\overline{\boldsymbol{D}}_{(11)} & \mathbf{0} & \overline{\boldsymbol{D}}_{(21)} \in \mathbb{R}^{r \times N-R} \\
\mathbf{0} & \mathbf{0} & \overline{\boldsymbol{D}}_{(22)} \in \mathbb{R}^{R-r \times N-R} \\
\mathbf{0} & \mathbf{0} & \overline{\boldsymbol{D}}_{(3)} \in \mathbb{R}^{N-R \times N-R}
\end{array}\right] \overline{\boldsymbol{H}}=\left[\begin{array}{c}
\overline{\boldsymbol{H}}_{(1)} \\
\overline{\boldsymbol{H}}_{(2)} \\
\mathbf{0}
\end{array}\right]
$$

where $\overline{\boldsymbol{H}}_{(1)} \in \mathbb{R}^{r \times L}$ and $\overline{\boldsymbol{H}}_{(2)} \in \mathbb{R}^{R-r \times L}$. Define $R_{s} \triangleq r+$ $\min \{s, R-r\}$. Then, the part of the state vector corresponding to the first $R_{s}$ entries is $s$-sparse-controllable, while the remaining part is $s$-sparse-uncontrollable. Also, since $\bar{D}=$ $(\boldsymbol{U} \boldsymbol{W})^{-1} \boldsymbol{D}(\boldsymbol{U} \boldsymbol{W})$ and $\overline{\boldsymbol{H}}=(\boldsymbol{U} \boldsymbol{W})^{-1} \boldsymbol{H}$, the new basis is $\boldsymbol{U} \boldsymbol{W}$.

Here, steps 1 and 2 are the same as the Kalman decomposition, and in steps 3 and 4, we find a basis that separates the sparse-controllable

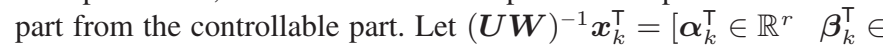


$\left.\mathbb{R}^{R-r} \quad \gamma_{k}^{\top} \in \mathbb{R}^{N-R}\right]$. We then have the following equations which are equivalent to (1):

$$
\begin{aligned}
\boldsymbol{\alpha}_{k} & =\overline{\boldsymbol{D}}_{(11)} \boldsymbol{\alpha}_{k-1}+\overline{\boldsymbol{D}}_{(21)} \boldsymbol{\gamma}_{k-1}+\overline{\boldsymbol{H}}_{(1)} \boldsymbol{h}_{k} \\
\boldsymbol{\beta}_{k} & =\overline{\boldsymbol{D}}_{(22)} \boldsymbol{\gamma}_{k-1}+\overline{\boldsymbol{H}}_{(2)} \boldsymbol{h}_{k} \\
\boldsymbol{\gamma}_{k} & =\overline{\boldsymbol{D}}_{(3)} \boldsymbol{\gamma}_{k-1} .
\end{aligned}
$$

Clearly, $\gamma_{k}$ is uncontrollable as it is independent of the input sequence. Further, the Kalman decomposition ensures that the part of the state vector corresponding to $\left[\begin{array}{ll}\boldsymbol{\alpha}_{k}^{\top} & \boldsymbol{\beta}_{k}^{\top}\end{array}\right]^{\top}$ is controllable. Thus

$$
\operatorname{Rank}\left\{\left[\begin{array}{ccc}
\overline{\boldsymbol{D}}_{(11)}-\lambda \boldsymbol{I} & \mathbf{0} & \overline{\boldsymbol{H}}_{(1)} \\
\mathbf{0} & \mathbf{0} & \overline{\boldsymbol{H}}_{(2)}
\end{array}\right]\right\}=R
$$

for any $\lambda \in \mathbb{C}$, and hence

$$
\operatorname{Rank}\left\{\left[\begin{array}{ccc}
\overline{\boldsymbol{D}}_{(11)}-\lambda \boldsymbol{I} & \mathbf{0} & \overline{\boldsymbol{H}}_{(1)} \\
\mathbf{0} & \mathbf{0} & \left(\overline{\boldsymbol{H}}_{(2)}^{\top}\right)_{\mathcal{S}}^{\top}
\end{array}\right]\right\}=r+|\mathcal{S}|
$$

for any index set $\mathcal{S} \subseteq[R-r]$. Therefore, from the inequality constraint of Theorem 1, choosing $|\mathcal{S}|=\min \{s, R-r\}$ ensures that the part of $\left[\begin{array}{ll}\boldsymbol{\alpha}_{k}^{\top} & \boldsymbol{\beta}_{k, \mathcal{S}}^{\top}\end{array}\right] \in \mathbb{R}^{R_{s}}$ corresponds to the sparse controllable part of the state vector. We choose $\mathcal{S}$ as the top $R_{s}-r$ indices of the new state vector. This is valid because $\alpha_{k}$ is independent of $\beta_{k-1}$, and $\beta_{k}$ is independent of both $\alpha_{k-1}$ and $\beta_{k-1}$.

\section{CONCLUSION}

We presented two easily verifiable necessary and sufficient conditions for controllability of linear systems subject to sparsity constraints on the input. Further, we bounded the minimum number of sparse input vectors that ensure controllability. The sparse-controllability tests led to a Kalman decomposition-like procedure for separating the system into sparse-controllable, controllable but sparse-uncontrollable, and uncontrollable parts. We also extended our results to the output controllability and controllability using sparse inputs with a common support. However, our work does not impose any constraint on the $\ell_{\infty}$ norm of the input vector, which may be required in applications where the maximum input magnitude is constrained. Addressing sparse-controllability under this constraint is an interesting avenue for future work.

\section{ACKNOWLEDGMENT}

We thank Chandrasekhar Sriram (Indian Institute of Science, Bangalore) for several helpful discussions.

\section{APPENDIX A}

\section{PROOF OF THEOREM 1}

Proof: We show that the conditions of the theorem are equivalent to the Kalman-type rank test. The proof relies on the fact that the Kalman rank test for the unconstrained system is equivalent to the PBH test, which is the same as the rank condition of Theorem 1 [2].

We first prove that conditions of Theorem 1 imply the Kalman-type rank test. Suppose that the Kalman-type rank test fails. Then, consider the following matrix of size $N \times N \tilde{K} s$ :

$$
\begin{array}{r}
\tilde{\boldsymbol{H}}^{*}=\left[\boldsymbol{D}^{\tilde{K} N-1} \boldsymbol{H}_{\mathcal{S}_{1}} \boldsymbol{D}^{\tilde{K} N-2} \boldsymbol{H}_{\mathcal{S}_{1}} \ldots \boldsymbol{D}^{(\tilde{K}-1) N} \boldsymbol{H}_{\mathcal{S}_{1}}\right. \\
\ldots \boldsymbol{D}^{(\tilde{K}-1) N-1} \boldsymbol{H}_{\mathcal{S}_{2}} \ldots \boldsymbol{D}^{(\tilde{K}-2) N} \boldsymbol{H}_{\mathcal{S}_{2}} \ldots \\
\left.\ldots \boldsymbol{D}^{N-1} \boldsymbol{H}_{\mathcal{S}_{\tilde{K}}} \ldots \boldsymbol{H}_{\mathcal{S}_{\tilde{K}}}\right]
\end{array}
$$

where we define $\tilde{K} \triangleq\lceil L / s\rceil$ index sets as follows:

$$
\left|\mathcal{S}_{i}\right|=s, \quad \cup_{i=1}^{\tilde{K}} \mathcal{S}_{i}=[L] .
$$

We note that $\tilde{\boldsymbol{H}}^{*}$ has the same form as that of the matrix for the Kalmantype rank test for sparse-controllability in (5), with $K$ as $N \tilde{K}$. Since the Kalman-type rank test fails, $\tilde{\boldsymbol{H}}^{*}$ does not have full row rank. Further, we can rearrange the columns of $\tilde{\boldsymbol{H}}^{*}$ to get the following matrix which has the same rank as that of $\tilde{\boldsymbol{H}}^{*}$ :

$$
\left[\begin{array}{llll}
\boldsymbol{D}^{N-1} \boldsymbol{H}^{*} & \boldsymbol{D}^{N-2} \boldsymbol{H}^{*} & \ldots & \boldsymbol{H}^{*}
\end{array}\right]
$$

where we define the matrix $\boldsymbol{H}^{*} \in \mathbb{R}^{N \times \tilde{K} s}$ as follows:

$$
\boldsymbol{H}^{*} \triangleq\left[\boldsymbol{D}^{(\tilde{K}-1) N} \boldsymbol{H}_{\mathcal{S}_{1}} \quad \boldsymbol{D}^{(\tilde{K}-2) N} \boldsymbol{H}_{\mathcal{S}_{2}} \ldots \boldsymbol{H}_{\mathcal{S}_{\tilde{K}}}\right] .
$$

Thus, using the classical Kalman rank test for the unconstrained inputs, the system defined by the matrix tuple $\left(\boldsymbol{D}, \boldsymbol{H}^{*}\right)$ is not controllable. Then, the classical PBH test for the unconstrained inputs implies that there exists $\lambda \in \mathbb{C}$ such that $\operatorname{Rank}\left\{\left[\boldsymbol{D}-\lambda \boldsymbol{I} \boldsymbol{H}^{*}\right]\right\}<N$. Therefore, there exists a nonzero vector $\boldsymbol{z} \in \mathbb{R}^{N}$ such that $\boldsymbol{z}^{\top} \boldsymbol{D}=\lambda \boldsymbol{z}^{\top}$ and $\boldsymbol{z}^{\top} \boldsymbol{H}^{*}=\mathbf{0}$. However, we have

$$
\mathbf{0}=\boldsymbol{z}^{\top} \boldsymbol{H}^{*}=\boldsymbol{z}^{\top}\left[\lambda^{(\tilde{K}-1) N} \boldsymbol{H}_{\mathcal{S}_{1}} \quad \lambda^{(\tilde{K}-2) N} \boldsymbol{H}_{\mathcal{S}_{2}} \ldots \quad \boldsymbol{H}_{\mathcal{S}_{\tilde{K}}}\right] .
$$

So either $\lambda=0$ and $\boldsymbol{z}^{\top} \boldsymbol{H}_{\mathcal{S}_{\tilde{K}}}=\mathbf{0}$, or, if $\lambda \neq 0, \boldsymbol{z}^{\top} \boldsymbol{H}=\mathbf{0}$ because $\boldsymbol{z}$ is orthogonal to all columns of $\boldsymbol{H}$ due to (23). Hence, for every index set $\mathcal{S}_{i}$ with $s$ entries, there exists $\boldsymbol{z} \in \mathbb{R}^{N}$ such that $\boldsymbol{z}^{\top} \boldsymbol{D}=\lambda \boldsymbol{z}^{\top}$, and either $\lambda=0$ and $\boldsymbol{z}^{\top} \boldsymbol{H}_{\mathcal{S}_{i}}=\mathbf{0}$, or $\boldsymbol{z}^{\top} \boldsymbol{H}=\mathbf{0}$. Therefore, one of the following cases hold.

1) There exists a left eigenvector $\boldsymbol{z}$ of $\boldsymbol{D}$, such that $\boldsymbol{z}^{\top} \boldsymbol{H}=\mathbf{0}$. Thus, the rank condition of Theorem 1 does not hold.

2) For every left eigenvector $\boldsymbol{z}$ of $\boldsymbol{D}$, we have $\boldsymbol{z}^{\top} \boldsymbol{H} \neq \mathbf{0}$. However, for every index set $\mathcal{S}$ with $s$ entries, there exists a nonzero vector $\boldsymbol{z} \in \mathbb{R}^{N}$ such that $\boldsymbol{z}^{\top} \boldsymbol{D}=\mathbf{0}$, and $\boldsymbol{z}^{\top} \boldsymbol{H}_{\mathcal{S}}=\mathbf{0}$. This implies that $\operatorname{Rank}\left\{\left[\begin{array}{ll}\boldsymbol{D} & \boldsymbol{H}\end{array}\right]\right\}=N$ and for every index set $\mathcal{S}$ with $s$ entries, there exists $\boldsymbol{z} \in \mathbb{R}^{N}$ such that $\boldsymbol{z}^{\top}\left[\begin{array}{ll}\boldsymbol{D} & \boldsymbol{H}_{\mathcal{S}}\end{array}\right]=\mathbf{0}$. Therefore, $s<N-R_{D} \leq R_{H}$. Thus, the inequality condition in Theorem 1 does not hold.

Thus, when the Kalman-type rank test is unsuccessful, the conditions of the theorem are also violated.

Next, we prove that the Kalman-type rank test implies the conditions of the theorem. Suppose that the two conditions do not hold simultaneously. This could happen under the following two exhaustive cases.

1) Suppose that the rank condition does not hold. Then, the PBH test is violated, which implies that the system is not controllable. Hence, it cannot be sparse-controllable.

2) Suppose that the rank condition holds, but the inequality condition does not hold. Then, for every index set $\mathcal{S}$ with $s$ entries, there exists a nonzero vector $\boldsymbol{z}$ such that $\boldsymbol{z}^{\top} \boldsymbol{H}_{\mathcal{S}}=\mathbf{0}$ and $\boldsymbol{z}^{\top} \boldsymbol{D}=\mathbf{0}$. This implies that for any set of $K>0$ index sets $\left\{\mathcal{S}_{i}:\left|\mathcal{S}_{i}\right|=s\right\}_{i=1}^{K}$ there exists a nonzero vector $\boldsymbol{z} \in \mathbb{R}^{N}$ such that

$$
\boldsymbol{z}^{\top}\left[\begin{array}{llll}
\boldsymbol{D}^{K-1} \boldsymbol{H}_{\mathcal{S}_{1}} & \boldsymbol{D}^{K-2} \boldsymbol{H}_{\mathcal{S}_{2}} & \ldots & \boldsymbol{H}_{\mathcal{S}_{K}}
\end{array}\right]=\mathbf{0} .
$$

Hence, the Kalman-type rank test for fails.

Thus, the proof is complete.

\section{APPENDIX B PROOF OF THEOREM 3}

Using the Kalman-type rank test, the minimum number of input vectors required to ensure controllability is the smallest integer $K$ that satisfies the rank condition of the test. So, for any finite $K$, we define 
$\mathcal{H}_{(K)} \subseteq \mathbb{R}^{N \times K s}$ as the set of submatrices of $\tilde{\boldsymbol{H}}_{(K)}$ of the form given in (5). Also, we define the following:

$$
\begin{aligned}
R_{(K)}^{*} & =\max _{\boldsymbol{H}_{(K)} \in \mathcal{H}_{(K)}} \operatorname{Rank}\left\{\boldsymbol{H}_{(K)}\right\} \\
\mathcal{H}_{(K)}^{*} & =\left\{\boldsymbol{H}_{(K)} \in \mathcal{H}_{(K)}: \operatorname{Rank}\left\{\boldsymbol{H}_{(K)}\right\}=R_{(K)}^{*}\right\} .
\end{aligned}
$$

With these definitions, $K^{*}$ is the smallest integer $K$ satisfying $R_{(K)}^{*}=$ $N$.

Before starting the proof, we outline the main steps involved. At a high level, there are five steps to the proof.

1) We begin by showing that for any matrix $\boldsymbol{H}_{(K)} \in \mathcal{H}_{(K)}$, we can find a matrix $\boldsymbol{H}_{(K)}^{*} \in \mathcal{H}_{(K)}^{*}$ such that

$$
\mathcal{C S}\left\{\boldsymbol{H}_{(K)}\right\} \subseteq \mathcal{C S}\left\{\boldsymbol{H}_{(K)}^{*}\right\}
$$

2) Second, using the above claim, we show that if $K$ is any integer such that

$$
R_{(K)}^{*}=R_{(K+1)}^{*}
$$

then $R_{(K+Q)}^{*}=R_{(K)}^{*}$, for any positive integer $Q$.

3) Third, we prove that $K^{*}$ is the smallest integer $K$ such that (29) holds, which in turn leads to the upper bound: $K^{*} \leq N+1-$ $R_{\boldsymbol{H}, s}^{*}$, where $R_{\boldsymbol{H}, s}^{*}$ is as defined in the statement of the theorem.

4) Fourth, we show that in order to satisfy the rank criterion in (29), $\boldsymbol{H}_{\left(K^{*}\right)}^{*}$ needs to contain at most $q S^{*}$ number of columns with a particular structure. Then, we provide a choice of index sets $\left\{\mathcal{S}_{i}\right\}_{i=1}^{K=q\left\lceil S^{*} / s\right\rceil}$ which can lead to that particular structure. Since the smallest integer $K$ that can meet the rank criterion in (29) is $K^{*}$, we assert that $K^{*} \leq q\left\lceil S^{*} / s\right\rceil$. Thus, together with the above step, we establish the upper bound in the theorem.

5) Finally, we lower bound $K^{*}$ to complete the proof.

\section{A. Characterizing $\mathcal{H}_{(K)}^{*}$}

If $\boldsymbol{H}_{(K)} \in \mathcal{H}_{(K)}^{*}$, the result is trivial: $\boldsymbol{H}_{(K)}^{*}=\boldsymbol{H}_{(K)}$. Suppose that $\boldsymbol{H}_{(K)} \notin \mathcal{H}_{(K)}^{*}$, then $\operatorname{Rank}\left\{\boldsymbol{H}_{(K)}\right\}<R_{(K)}^{*}$. Therefore, to find $\boldsymbol{H}_{(K)}^{*}$, we have to replace some linearly dependent columns of $\boldsymbol{H}_{(K)}$ with columns which are linearly independent of the rest of the columns of $\boldsymbol{H}_{(K)}$, as follows.

1) Find a set $\left\{\boldsymbol{u}_{i}\right\}_{i=1}^{\operatorname{Rank}\left\{\boldsymbol{H}_{(K)}\right\}}$ of columns of $\boldsymbol{H}_{(K)}$ that are linearly independent and span $\mathcal{C S}\left\{\boldsymbol{H}_{(K)}\right\}$.

2) Since $\boldsymbol{H}_{(K)}$ is a submatrix of $\tilde{\boldsymbol{H}}_{(K)}$, we can extend the set $\left\{\boldsymbol{u}_{i}\right\}_{i=1}^{\operatorname{Rank}\left\{\boldsymbol{H}_{(K)}\right\}}$ to form a basis $\left\{\boldsymbol{u}_{i}\right\}_{i=1}^{\operatorname{Rank}\left\{\tilde{\boldsymbol{H}}_{(K)}\right\}}$ of $\mathcal{C S}\left\{\tilde{\boldsymbol{H}}_{(K)}\right\}$ by adding columns from $\tilde{\boldsymbol{H}}_{(K)}$. We note that $\boldsymbol{u}_{i}=\boldsymbol{D}^{p} \boldsymbol{H}_{j}$ for some integers $p$ and $j$ because of the structure of $\tilde{\boldsymbol{H}}_{(K)}$.

3) Replace the linearly dependent columns of $\boldsymbol{H}_{(K)}$ with the columns from the set $\left\{\boldsymbol{u}_{i}\right\}_{i=\operatorname{Rank}\left\{\boldsymbol{H}_{(K)}\right\}+1}^{\operatorname{Rank}\left\{\tilde{\boldsymbol{H}}_{(K)}\right\}}$ to get a new matrix $\overline{\boldsymbol{H}}_{(K)} \in$ $\mathbb{R}^{N \times K s}$. We only replace a column of the form $\boldsymbol{D}^{p} \boldsymbol{H}_{j}$ in $\boldsymbol{H}_{(K)}$ with another column of the form $\boldsymbol{D}^{p} \boldsymbol{H}_{j^{\prime}}$, for all $p$ and $j$ and some integer $j^{\prime}$. This ensures that $\overline{\boldsymbol{H}}_{(K)} \in \mathcal{H}_{(K)}$. In this fashion, we replace as many columns of $\boldsymbol{H}_{(K)}$ as necessary to ensure that $\overline{\boldsymbol{H}}_{(K)}$ has the maximum rank, $R_{(K)}^{*}$. However, since we are only replacing linearly dependent columns, we have

$$
\mathcal{C S}\left\{\boldsymbol{H}_{(K)}\right\} \subseteq \mathcal{C S}\left\{\overline{\boldsymbol{H}}_{(K)}\right\} .
$$

Since $\operatorname{Rank}\left\{\overline{\boldsymbol{H}}_{(K)}\right\}=R_{(K)}^{*}$ and $\overline{\boldsymbol{H}}_{(K)} \in \mathcal{H}_{(K)}$, we get that $\overline{\boldsymbol{H}}_{(K)} \in \mathcal{H}_{(K)}^{*}$, satisfying (30). Hence, the first step of the proof is complete.

\section{B. Characterizing $R_{(K)}^{*}$}

We use induction to show that $R_{(K+Q)}^{*}=R_{(K)}^{*}$, for any integer $Q>0$. Hence, it suffices to show the following:

$$
R_{(K+2)}^{*}=R_{(K+1)}^{*} .
$$

From (26), we know that $R_{(K+2)}^{*} \geq R_{(K+1)}^{*}$. Also

$$
R_{(K)}^{*}=\max _{\boldsymbol{H}_{(K)} \in \mathcal{H}_{(K)}} \operatorname{dim}\left\{\mathcal{C S}\left\{\boldsymbol{H}_{(K)}\right\}\right\}
$$

where $\operatorname{dim}\{\cdot\}$ denotes the dimension of a subspace. Thus, we establish (31) by showing that for any matrix $\boldsymbol{H}_{(K+2)} \in \mathcal{H}_{(K+2)}$, there exists a matrix $\boldsymbol{H}_{(K+1)}^{*} \in \mathcal{H}_{(K+1)}^{*}$ such that

$$
\mathcal{C S}\left\{\boldsymbol{H}_{(K+2)}\right\} \subseteq \mathcal{C S}\left\{\boldsymbol{H}_{(K+1)}^{*}\right\} .
$$

We prove this relation by separately looking at the column spaces spanned by the first $s$ columns and the last $(K+1) s$ columns of $\boldsymbol{H}_{(K+2)}$. We know that the submatrix formed by the last $(K+1) s$ columns of any matrix in $\mathcal{H}_{(K+2)}$ belongs to $\mathcal{H}_{(K+1)}$. Thus, using the claim in the first step, we can find a matrix $\boldsymbol{H}_{(K+1)}^{*}$ such that the column space spanned by the last $(K+1) s$ columns of $\boldsymbol{H}_{(K)}$ is contained in $\mathcal{C} \mathcal{S}\left\{\boldsymbol{H}_{(K+1)}^{*}\right\}$. Therefore, it suffices to show that the column space spanned by the first $s$ columns of $\boldsymbol{H}_{(K+2)}$ is contained in the column space of $\boldsymbol{H}_{(K+1)}^{*}$.

To prove the above statement, we note that the column space of the first $s$ columns of $\boldsymbol{H}_{(K+2)}$ is contained in $\mathcal{C S}\left\{\boldsymbol{D}^{K+1} \boldsymbol{H}\right\}$. Also, $\mathcal{C S}\left\{\boldsymbol{H}_{(K+1)}^{*}\right\}$ contains $\cap_{\boldsymbol{H}_{(K+1)}^{*} \in \mathcal{H}_{(K+1)}^{*}} \mathcal{C S}\left\{\boldsymbol{H}_{(K+1)}^{*}\right\}$. Hence, it suffices to show that

$$
\mathcal{C S}\left\{\boldsymbol{D}^{K+1} \boldsymbol{H}\right\} \subseteq \boldsymbol{H}_{(K+1)}^{*} \in \cap_{(K+1)} \mathcal{C S}\left\{\boldsymbol{H}_{(K+1)}^{*}\right\}
$$

which we prove using the relation (29).

To show that (34) holds, we consider an index set $\mathcal{S} \subseteq[L]$ with $s$ en-

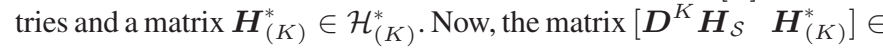
$\mathbb{R}^{N \times(K+1) s}$ belongs to $\mathcal{H}_{(K+1)}$. Thus, from (26) and (29) we have

$$
\operatorname{Rank}\left\{\left[\begin{array}{ll}
D^{K} \boldsymbol{H}_{\mathcal{S}} & \boldsymbol{H}_{(K)}^{*}
\end{array}\right]\right\} \leq R_{(K+1)}^{*}=R_{(K)}^{*} .
$$

However, we also have

$$
\operatorname{Rank}\left\{\left[\begin{array}{ll}
\boldsymbol{D}^{K} \boldsymbol{H}_{\mathcal{S}} & \boldsymbol{H}_{(K)}^{*}
\end{array}\right]\right\} \geq \operatorname{Rank}\left\{\boldsymbol{H}_{(K)}^{*}\right\}=R_{(K)}^{*} .
$$

Thus, for all index sets $\mathcal{S}$ with $s$ entries and any $\boldsymbol{H}_{(K)}^{*} \in \mathcal{H}_{(K)}^{*}$

$$
\operatorname{Rank}\left\{\left[\begin{array}{ll}
\boldsymbol{D}^{K} \boldsymbol{H}_{\mathcal{S}} & \boldsymbol{H}_{(K)}^{*}
\end{array}\right]\right\}=\operatorname{Rank}\left\{\boldsymbol{H}_{(K)}^{*}\right\}
$$

This relation immediately implies the following:

$$
\operatorname{Rank}\left\{\left[\begin{array}{ll}
\boldsymbol{D}^{K} \boldsymbol{H} & \boldsymbol{H}_{(K)}^{*}
\end{array}\right]\right\}=\operatorname{Rank}\left\{\boldsymbol{H}_{(K)}^{*}\right\}
$$

for any matrix $\boldsymbol{H}_{(K)}^{*} \in \mathcal{H}_{(K)}^{*}$. Thus, we get that the columns of $\boldsymbol{D}^{K} \boldsymbol{H}$ belong to $\mathcal{C S}\left\{\boldsymbol{H}_{(K)}^{*}\right\}$, for any matrix $\boldsymbol{H}_{(K)}^{*} \in \mathcal{H}_{(K)}^{*}$. Hence

$$
\mathcal{C S}\left\{\boldsymbol{D}^{K} \boldsymbol{H}\right\} \subseteq{ }_{\boldsymbol{H}_{(K)}^{*}} \cap_{\mathcal{H}_{(K)}^{*}} \mathcal{C S}\left\{\boldsymbol{H}_{(K)}^{*}\right\} .
$$

Therefore, we get

$$
\mathcal{C S}\left\{\boldsymbol{D}^{K+1} \boldsymbol{H}\right\} \subseteq \boldsymbol{H}_{(K)}^{*} \in \cap_{(K)}^{*} \mathcal{C S}\left\{\boldsymbol{D} \boldsymbol{H}_{(K)}^{*}\right\} .
$$


Hence, to prove (34), we need to show that

$$
\boldsymbol{H}_{(K)}^{*} \in \mathcal{H}_{(K)}^{*} \mathcal{C S}\left\{\boldsymbol{D} \boldsymbol{H}_{(K)}^{*}\right\} \subseteq \overbrace{\boldsymbol{H}_{(K+1)}^{*} \in \mathcal{H}_{(K+1)}^{*}} \mathcal{C S}\left\{\boldsymbol{H}_{(K+1)}^{*}\right\} .
$$

We prove the above relation by showing that there exists a matrix $\boldsymbol{H}_{(K+1)}^{*} \in \mathcal{H}_{(K+1)}^{*}$ such that

$$
\mathcal{C S}\left\{\boldsymbol{D} \boldsymbol{H}_{(K)}^{*}\right\} \subseteq \mathcal{C S}\left\{\boldsymbol{H}_{(K+1)}^{*}\right\}
$$

for every matrix $\boldsymbol{H}_{(K)}^{*} \in \mathcal{H}_{(K)}^{*}$. So we consider a new matrix $\overline{\boldsymbol{H}}_{(K+1)} \in \mathbb{R}^{N \times(K+1) s}$ as follows:

$$
\overline{\boldsymbol{H}}_{(K+1)} \triangleq\left[\begin{array}{ll}
\boldsymbol{D} \boldsymbol{H}_{(K)}^{*} & \boldsymbol{H}_{\mathcal{S}}
\end{array}\right]
$$

for some index set $\mathcal{S} \subseteq[L]$ and $|\mathcal{S}|=s$. Since $\overline{\boldsymbol{H}}_{(K+1)} \in \mathcal{H}_{(K+1)}$, using the arguments in the first step, we can find a matrix $\boldsymbol{H}_{(K+1)}^{*} \in$ $\mathcal{H}_{(K+1)}^{*}$ such that

$$
\mathcal{C S}\left\{\overline{\boldsymbol{H}}_{(K+1)}\right\} \subseteq \mathcal{C S}\left\{\boldsymbol{H}_{(K+1)}^{*}\right\}
$$

However, (43) implies that $\mathcal{C S}\left\{\boldsymbol{D H}_{(K)}^{*}\right\} \subseteq \mathcal{C S}\left\{\overline{\boldsymbol{H}}_{(K+1)}\right\}$. Therefore, (42) holds, and hence (41) is proved.

Recall that (41) implies (34), which in turn establishes the relation (31). By mathematical induction, we conclude that $\operatorname{Rank}\left\{\boldsymbol{H}_{(K+Q)}^{*}\right\}=$ $\operatorname{Rank}\left\{\boldsymbol{H}_{(K)}^{*}\right\}$, for any positive integer $Q$, and the proof of the second step in the outline is complete.

\section{First Part of the Upper Bound}

Suppose that $K_{*}$ is the smallest integer such that $R_{\left(K_{*}\right)}^{*}=R_{\left(K_{*}+1\right)}^{*}$ From (26), it is clear that

$$
R_{(K)}^{*} \leq R_{(K+1)}^{*} \leq N
$$

for any positive integer $K$. Since $R_{\left(K^{*}\right)}^{*}=N$, we have $R_{\left(K^{*}\right)}^{*}=$ $R_{\left(K^{*}+1\right)}^{*}=N$. Therefore, $K_{*} \leq K^{*}$, and $R_{\left(K_{*}\right)}^{*}=N$ from the claim in the second step.

Further, since $K^{*}$ is the smallest integer such that $R_{\left(K^{*}\right)}^{*}=N$, we have $K_{*}=K^{*}$. Hence, $R_{(K)}^{*}$ strictly increases with $K$, for $1 \leq K \leq$ $K^{*}$, and we have

$$
\begin{aligned}
N & =R_{\left(K^{*}\right)}^{*} \geq R_{\left(K^{*}-1\right)}^{*}+1 \geq R_{\left(K^{*}-2\right)}^{*}+2 \\
& \geq R_{(1)}^{*}+K^{*}-1=R_{\boldsymbol{H}, s}^{*}+K^{*}-1 .
\end{aligned}
$$

Hence, the third step in the outline is complete.

\section{Upper Bounding $K^{*}$}

To prove that $K^{*} \leq q\left\lceil S^{*} / s\right\rceil$, we first look at the linearly independent columns in $\boldsymbol{H}_{\left(K^{*}\right)}^{*}$. For any $K$, each column of $\boldsymbol{H}_{(K)}^{*}$ is of the form $\boldsymbol{D}^{p} \boldsymbol{H}_{j}$, for some integer $p$, and $j \in[L]$. However, since $q$ is the degree of the minimal polynomial of $\boldsymbol{D}$, for any integer $Q, \boldsymbol{D}^{p}$ can be expressed as a linear combination of $\left\{\boldsymbol{D}^{i}\right\}_{i=Q}^{Q+q-1}$, for all $p \geq Q$. Therefore, for any $j$, if $\left\{\boldsymbol{D}^{i} \boldsymbol{H}_{j} \in \mathbb{R}^{N}\right\}_{i=Q}^{Q+q-1}$ are any $q$ columns of $\boldsymbol{H}_{(K)}^{*}$, further adding columns of the form $\boldsymbol{D}^{p} \boldsymbol{H}_{j}, p \geq Q$, does not improve the rank of the matrix. Therefore, for a given $j$, at most $q$ columns of the form $\boldsymbol{D}^{p} \boldsymbol{H}_{j}$ need to be present in $\boldsymbol{H}_{(K)}^{*}$ to ensure the rank criterion in (29).

Further, let $\boldsymbol{H}_{\mathcal{S}^{\prime}}$ with $\mathcal{S}^{\prime} \subseteq[L]$ represent the smallest set of columns of $\boldsymbol{H}$ such that the linear system described by the tuple $\left(\boldsymbol{D}, \boldsymbol{H}_{\mathcal{S}}\right)$ is controllable. As given in the statement of the theorem, let $S^{*}=\left|\mathcal{S}^{\prime}\right|$. Then, for any integer $p$, if $\left\{\boldsymbol{D}^{p} \boldsymbol{H}_{j} \in \mathbb{R}^{N}\right\}_{j \in \mathcal{S}^{\prime}}$ are any $S^{*}$ columns of $\boldsymbol{H}_{(K)}^{*}$, further adding columns of the form $\boldsymbol{D}^{p} \boldsymbol{H}_{j}$, for $j \notin \mathcal{S}^{\prime}$ does not improve the rank of the matrix. Thus, for any given $p$, at most $S^{*}$ columns of the form $\boldsymbol{D}^{p} \boldsymbol{H}_{j}$ need to be present in $\boldsymbol{H}_{(K)}^{*}$ to ensure the rank criterion.

In short, we have proved that, in order to ensure the rank criterion in (29), $\boldsymbol{H}_{(K)}^{*}$ needs to have at most $q$ columns of the form $\boldsymbol{D}^{p} \boldsymbol{H}_{j}$, for any given $j$, and at most $S^{*}$ columns of the form $\boldsymbol{D}^{p} \boldsymbol{H}_{j}$, for any given $p$. Hence, $\boldsymbol{H}_{(K)}^{*}$ needs to have at most $q S^{*}$ columns to satisfy the rank criterion in (29).

Finally, we provide a choice of index sets for each input vector, that satisfies the above conditions. We form index sets $\left\{\mathcal{S}_{i}^{\prime}\right\}_{i=1}^{K=\left\lceil S^{*} / s\right\rceil}$ that partition the set of $S^{*}$ columns into groups of size at most $s$. The index sets are selected such that $\cup_{i=1}^{K} \mathcal{S}_{i}^{\prime}=\mathcal{S}^{\prime},\left|\mathcal{S}_{i}\right|=s$, and $\mathcal{S}_{K}$ is such that $\left[\begin{array}{ll}\boldsymbol{D} & \boldsymbol{H}_{\mathcal{S}_{K}}\end{array}\right]$ has rank $N$. The existence of such an index set $\mathcal{S}_{K}$ is ensured by Theorem 1, and they need not be disjoint. Next, we choose $\mathcal{S}_{i}=\mathcal{S}_{j}^{\prime}$, for $i=(j-1) q+$ $1,(j-1) q+2, \ldots, j q$. Hence, we get the following submatrix of $\tilde{\boldsymbol{H}}_{(K)} \in \mathbb{R}^{N \times q K L}$ :

$$
\begin{array}{r}
\boldsymbol{H}_{(K)}^{*}=\left[\boldsymbol{D}^{K q-1} \boldsymbol{H}_{\mathcal{S}_{1}} \boldsymbol{D}^{K q-2} \boldsymbol{H}_{\mathcal{S}_{1}} \ldots \boldsymbol{D}^{(K-1) q} \boldsymbol{H}_{\mathcal{S}_{1}}\right. \\
\ldots \boldsymbol{D}^{(K-1) q-1} \boldsymbol{H}_{\mathcal{S}_{2}} \ldots \boldsymbol{D}^{(K-2) q} \boldsymbol{H}_{\mathcal{S}_{2}} \ldots \\
\left.\ldots \boldsymbol{D}^{q-1} \boldsymbol{H}_{\mathcal{S}_{K}} \ldots \boldsymbol{H}_{\mathcal{S}_{K}}\right] .
\end{array}
$$

It is easy to see that this choice of index sets ensures that for any given $p, S^{*}$ columns of the form $\boldsymbol{D}^{p} \boldsymbol{H}_{j}$ are present in $\boldsymbol{H}_{(K)}^{*}$. Also, for any given $j \in \mathcal{S}^{\prime}, q$ columns of $\left\{\boldsymbol{D}^{i} \boldsymbol{H}_{j} \in \mathbb{R}^{N}\right\}_{i=Q}^{Q+q-1}$ are present in $\boldsymbol{H}_{(K)}^{*}$. Hence, $K^{*} \leq q\left\lceil S^{*} / s\right\rceil$, which establishes the upper bound in (12).

\section{E. Lower Bounding $K^{*}$}

The lower bound is achieved when all columns of $\boldsymbol{H}_{(K)}^{*}$ are linearly independent. Thus, to ensure that $\operatorname{rank} \boldsymbol{H}_{(K)}^{*}$ is $N, K s \geq N$. However, if $s \geq R_{\boldsymbol{H}}$, the maximum number of independent columns become $K R_{\boldsymbol{H}}$, and thus we get that $K R_{\boldsymbol{H}} \geq N$. Hence, $K \min \left\{R_{\boldsymbol{H}}, s\right\} \leq N$, and the lower bound in (12) is proved.

As noted in the proof outline, this suffices to establish Theorem 3 .

\section{REFERENCES}

[1] R. Kalman, "On the general theory of control systems," IRE Trans. Autom. Control, vol. 4, no. 3, pp. 110-110, Dec. 1959.

[2] M. Hautus, "Stabilization controllability and observability of linear autonomous systems," in Proc. Indagationes Mathematicae, Jan. 1970, vol. 73, pp. 448-455.

[3] M. Nagahara and D. E. Quevedo, "Sparse representations for packetized predictive networked control," IFAC Proc. Vol., vol. 44, no. 1, pp. 84-89, Jan. 2011

[4] Z. Li, Y. Xu, H. Huang, and S. Misra, "Sparse control and compressed sensing in networked switched systems," IET Control Theory Appl., vol. 10, no. 9, pp. 1078-1087, Jun. 2016

[5] A. Jadbabaie, A. Olshevsky, and M. Siami, "Deterministic and randomized actuator scheduling with guaranteed performance bounds," May 2018, arXiv: 1805.00606.

[6] P. V. Chanekar, N. Chopra, and S. Azarm, "Optimal actuator placement for linear systems with limited number of actuators," in Proc. Amer. Control Conf., May 2017, pp. 334-339.

[7] E. Nozari, F. Pasqualetti, and J. Cortés, "Time-invariant versus timevarying actuator scheduling in complex networks," in Proc. Amer. Control Conf., May 2017, pp. 4995-5000.

[8] A. Olshevsky, "Minimal controllability problems," IEEE Trans. Control Netw. Syst., vol. 1, no. 3, pp. 249-258, Sep. 2014.

[9] V. Tzoumas, M. A. Rahimian, G. J. Pappas, and A. Jadbabaie, "Minimal actuator placement with bounds on control effort," IEEE Trans. Control Netw. Syst., vol. 3, no. 1, pp. 67-78, Mar. 2016. 
[10] Z. Liu et al., "Minimal input selection for robust control," CoRR, vol. abs/1712.01232, 2017. [Online]. Available: http://arxiv.org/abs/1712. 01232

[11] A. S. Charles, H. L. Yap, and C. J. Rozell, "Short-term memory capacity in networks via the restricted isometry property," Neural Comput., vol. 26, no. 6, pp. 1198-1235, Jun. 2014.

[12] S. Sefati, N. J. Cowan, and R. Vidal, "Linear systems with sparse inputs: Observability and input recovery," in Proc. Amer. Control Conf., Jul. 2015, pp. 5251-5257.

[13] M. Kafashan, A. Nandi, and S. Ching, "Relating observability and compressed sensing of time-varying signals in recurrent linear networks," Neural Netw., vol. 83, pp. 11-20, Nov. 2016.

[14] G. Joseph and C. R. Murthy, "On the observability of a linear system with a sparse initial state," IEEE Signal Process. Lett., vol. 25, no. 7, pp. 994-998, Jul. 2018.
[15] G. Joseph and C. R. Murthy, "Measurement bounds for observability of linear dynamical systems under sparsity constraints," IEEE Trans. Signal Process., vol. 67, no. 8, pp. 1992-2006, Feb. 2019.

[16] G. Joseph and C. R. Murthy, "Controllability of linear dynamical systems under input sparsity constraints," Dec. 2019. [Online]. Available: https: //arxiv.org/abs/1912.12224

[17] L. C. Westphal, Handbook of Control Systems Engineering. Berlin, Germany: Springer, 2012

[18] F. E. Hohn, Elementary Matrix Algebra. Chelmsford, MA, USA: Courier Corporation, 2013

[19] C.-T. Chen, Linear System Theory and Design. New York, NY, USA: Oxford Univ. Press, 1998

[20] R. E. Kalman, "Mathematical description of linear dynamical systems," SIAM J. Control, vol. 1, no. 2, pp. 152-192, 1963. 